\title{
Variation of the carrier-envelope phase of few-cycle laser pulses owing to the Gouy phase: a solid-state-based measurement
}

\author{
T. Tritschler, K. D. Hof, M. W. Klein, and M. Wegener \\ Institut für Angewandte Physik, Universität Karlsruhe (TH), Wolfgang-Gaede-Strasse 1, 76131 Karlsruhe, Germany
}

Received September 3, 2004

\begin{abstract}
The carrier-envelope phase of a laser pulse has recently become an important quantity in extreme nonlinear optics. Because of the topological Gouy phase, it changes while the pulse propagates through the focus of a lens. This variation is measured by a simple solid-state-based approach. The experimental results are analyzed by comparison with simple analytical model calculations. (C) 2005 Optical Society of America OCIS codes: $190.7110,300.6470$.
\end{abstract}

When focusing a monochromatic laser by means of a lens, the light field acquires a phase with respect to a plane wave while propagating along the optical axis $(z)$. This Gouy phase ${ }^{1-3}$ has been known for more than a century. Mathematically, ${ }^{4}$ it can be interpreted as a topological phase in analogy with the Berry phase ${ }^{5}$ for electrons. An intuitive discussion can be found in Ref. 6 . Propagating from $-\infty$ through the focus at $z=0$ to $+\infty$, the wave acquires a $\pi$ phase shift relative to a plane wave, which means that the carrier wave propagates from $-\infty$ to $+\infty$ faster than a plane wave by half a cycle of light-the phase velocity is superluminal, especially near the focus. On the other hand, the average group velocity of a laser pulse, i.e., the average velocity of the envelope, is luminal. Thus the carrier-envelope phase of a pulse changes while propagating through the focus. This effect becomes relevant in extreme nonlinear optics ${ }^{7}$ in which the carrier-envelope phase generally plays an important role.

The $\pi$ phase shift caused by the Gouy phase was directly time resolved in the terahertz regime a few years ago. ${ }^{8,9}$ More recently, ${ }^{10}$ the variation of the carrier-envelope phase versus the propagation coordinate was measured in the optical regime with an amplified femtosecond laser system and a sophisticated analysis of photoelectron spectra obtained from atoms ionized by the intense laser radiation within the focus. Nevertheless, it would still be interesting generally to have a simpler and specifically to have a solid-state-based approach suitable for measuring the shift of the carrier-envelope phase originating from the Gouy phase.

In this Letter we present corresponding experiments with a phase-frequency-stabilized 5 -fs laser oscillator and the recently introduced monolithic carrier-envelope-frequency detection by means of $\mathrm{ZnO}$ crystals in the perturbative regime. The experimental results are compared with calculations of the carrier-envelope phase based on the Gouy phase for different focusing conditions.

$\mathrm{ZnO}$ crystals indeed play a double role in the experiment (see Fig. 1). A fraction $(\geqslant 40 \mathrm{~mW})$ of the total average output power $(140-180 \mathrm{~mW})$ of our 5 -fs prism-based laser oscillator (a copy of that described in Ref. 11) operating at an $f_{r}=81 \mathrm{MHz}$ repetition frequency is tightly focused onto a first $250-\mu \mathrm{m}$-thick $\mathrm{ZnO}$ crystal with a linear polarization parallel to the crystallographic $\vec{c}$ axis $^{12}$ (which has no center of inversion). The beat signal arising from the interference of self-phase modulation and the second harmonic, both generated within the $\mathrm{ZnO}$ crystal, is selected by means of optical filters (3-mm Schott BG 39, 3-mm Schott GG 455, and Coherent 35-5289-000 $500-\mathrm{nm}$ short-pass interference filter) and converted into a voltage signal by a $50-\Omega$-terminated photomultiplier tube (Hamamatsu R4332, Bialkali). This carrier-envelope-frequency signal is fed into a feedback loop that controls the angle of the end mirror in the prism arm of the laser cavity by means of a piezoelectric actuator with an approximately $1-\mathrm{kHz}$ bandwidth. This allows us to stabilize the carrier-envelope frequency to $f_{\phi}=5 \mathrm{MHz}$, which is routinely monitored with a rf spectrum analyzer (Agilent PSA E4440A). The amplified and filtered photomultiplier signal is additionally used as a reference for a rf lock-in amplifier (Stanford Research Systems SR844).

The remaining fraction of the laser output power is attenuated by means of homemade metal-coated $100-\mu \mathrm{m}$-thin glass slides to an average power of

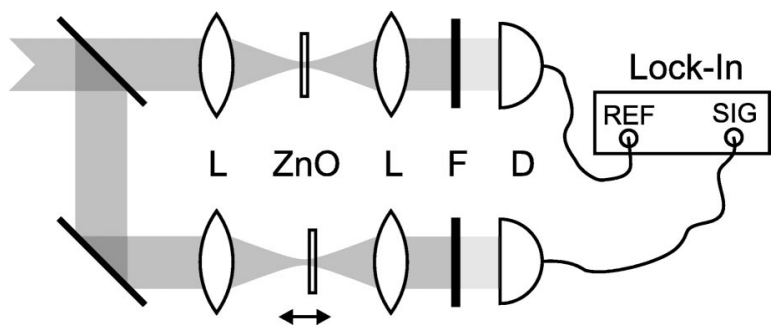

Fig. 1. Scheme of the experimental setup. The output of a 5 -fs mode-locked laser oscillator is split into two balanced arms. In each arm the light is focused with a Cassegrain lens (L) onto a $\mathrm{ZnO}$ crystal, followed by a set of optical filters (F), and a detector (D). One arm is used to stabilize the carrier-envelope frequency of the laser and additionally serves as the reference (REF) input for a lock-in amplifier. The other arm delivers the signal (SIG) input for the lock-in amplifier. The amplitude and phase of the output of the lock-in amplifier are recorded while scanning the $\mathrm{ZnO}$ crystal of the signal arm through the focus. 
$P=6 \mathrm{~mW}$, which is focused onto another 250 - $\mu$ m-thick $\mathrm{ZnO}$ crystal by a Cassegrainian lens (a mirror pair) with a focal length of $5.41 \mathrm{~mm}$ and a numerical aperture of 0.5 (Coherent 25-0522). The light emitted in the forward direction is collected by another Cassegrainian lens (13.41-mm focal length, numerical aperture 0.5, Coherent 25-0555), spectrally filtered as for the reference signal, sent to another $50-\Omega$-terminated photomultiplier tube (Hamamatsu R4332, Bialkali), and fed into the signal input of the rf lock-in amplifier. Importantly, the lock-in allows for measuring the amplitude and the phase of the $\mathrm{rf}$ signal with respect to the rf reference. ${ }^{13}$ It is clear that the rf lock-in phase in this arrangement directly reflects the shift of the carrier-envelope phase of the laser pulses-apart from an unknown but constant phase offset owing to the electronics and imperfect balancing of the two arms.

Figure 2(a) shows the beam width $w\left(z, \omega_{0}\right)$ of a fundamental Gaussian beam at laser carrier frequency $\omega_{0}$ (with $\hbar \omega_{0}=1.5 \mathrm{eV}$ ) within the Fresnel approximation $^{3}$ according to

$$
w^{2}(z, \omega)=w_{0}^{2}(\omega)\left\{1+\left[\frac{z}{z_{R}(\omega)}\right]^{2}\right\} .
$$

Here $w(0, \omega)=w_{0}(\omega)$ is the beam waist in the focal plane at $z=0$, and

$$
z_{R}(\omega)=\frac{w_{0}^{2}(\omega)}{2 c_{0}} \omega
$$

is the Rayleigh length at frequency $\omega$. The experimental Rayleigh length $z_{R}\left(\omega_{0}\right)$ can be obtained in two

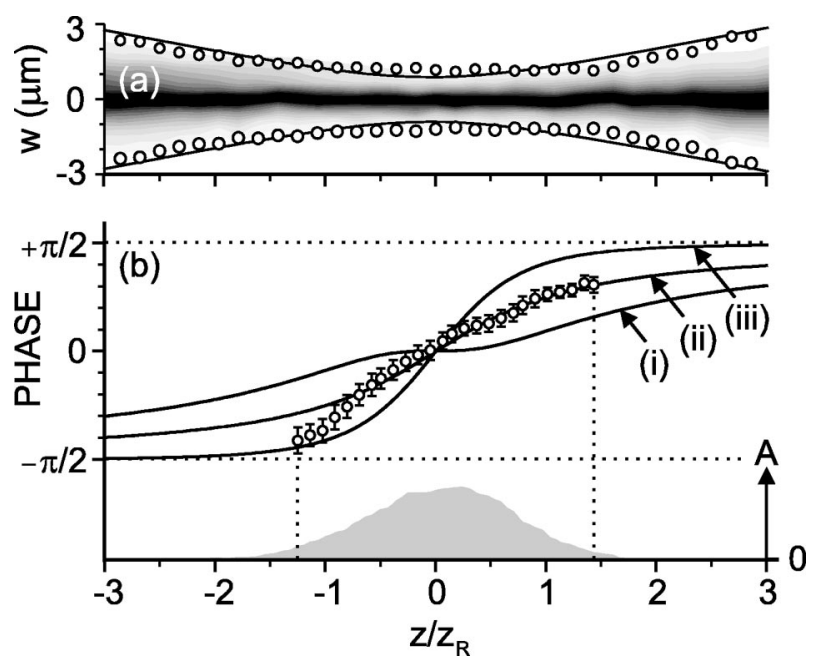

Fig. 2. (a) Gaussian beam width $w\left(z, \omega_{0}\right)$ versus propagation coordinate $z$ in units of the Rayleigh length $z_{R}\left(\omega_{0}\right)$ $=2.8 \mu \mathrm{m}$ at laser carrier frequency $\omega_{0}$. The open circles are the result of a knife-edge measurement. The corresponding raw data are shown by the gray-scale plot in the background. Here $50 \%$ of the maximum is set to black and $0 \%$ and $100 \%$ are set to white. (b) Lock-in phase (open circles), which is identical to the shift of carrier-envelope phase $\phi(z)$. The solid curves correspond to the theoretical expectation for cases (i)-(iii). Case (ii) is identical to the Gouy phase. Amplitude A of the lock-in signal is shown by the gray area at the bottom. different and independent ways. Both are based on a least-squares fit [solid curves in Fig. 2(a)] of $w\left(z, \omega_{0}\right)$ to the experimental data [open circles in Fig. 2(a)] that are obtained from a knife-edge measurement along the vertical direction. This fit delivers the two fit parameters $w_{0}\left(\omega_{0}\right)=0.9 \mu \mathrm{m}$ and $z_{R}\left(\omega_{0}\right)=2.8 \mu \mathrm{m}$ directly. As a consistency check, $z_{R}\left(\omega_{0}\right)$ can also be calculated with the above formula, inserting the value of $w_{0}\left(\omega_{0}\right)$ obtained from the fit. This delivers $z_{R}\left(\omega_{0}\right)$ $=3.0 \mu \mathrm{m}$, which is compatible with the above value within the experimental errors. From a knife-edge measurement along the horizontal direction we obtain $z_{R}\left(\omega_{0}\right)=2.4 \mu \mathrm{m}$. The curve labeled (ii) in Fig. 2(b) is the resulting Gouy phase $\varphi_{G}\left(z, \omega_{0}\right)$ according to

$$
\varphi_{G}(z, \omega)=\arctan \left[\frac{z}{z_{R}(\omega)}\right] .
$$

For higher-order modes a different prefactor results. ${ }^{6}$ The open circles in Fig. 2(b) correspond to the measured lock-in phase for variation of position $z$ of the $\mathrm{ZnO}$ crystal while keeping the Cassegrainian lenses fixed. $z>0$ means that the front of the $\mathrm{ZnO}$ crystal is behind the focus. The error bars reflect the statistical error of the average over ten individual scans. We depict only the phase in the central region of approximately $\pm 4 \mu \mathrm{m}$ in which the lock-in amplitude signal (gray area) has appreciable strength. In this region the phase increases monotonically. This might seem surprising at first considering that the $\mathrm{ZnO}$ crystal is more than an order of magnitude thicker than the Rayleigh length; hence a substantial averaging with respect to the phase should arise. However, the coherence length for phase matching of secondharmonic generation within the $\mathrm{ZnO}$ crystal can easily be estimated to be of the order of $1 \mu \mathrm{m}$ on the basis of the known Sellmeier coefficients for $\mathrm{ZnO}{ }^{14}$ Thus the effective sample thickness is indeed smaller than the Rayleigh length. We briefly note that a more complex behavior of the phase versus $z$ is observed at elevated powers incident onto the $\mathrm{ZnO}$ crystal of the signal arm (also see Ref. 15). The data shown in Fig. 2 are in the low-intensity limit.

We now discuss the relation between the measured carrier-envelope phase [Fig. 2(b)] and the Gouy phase shift. Generally, the carrier-envelope phase $\phi(z)$ of the laser electric field moving in the $+z$ direction, quoted at a fixed position $z$ on the optical axis,

$$
E(t)=\widetilde{E}(t) \cos \left[\omega_{0} t+\phi(z)\right],
$$

is determined by the Gouy phase but it is not identical to the Gouy phase. It results from the frequency dependence of the beam width $w\left(z_{f}, \omega\right)$ in front of the (ideal) lens at a focal length of $z=z_{f} \gg z_{R}\left(\omega_{0}\right)$. This is an experimentally known fact ${ }^{16,17}$ that translates into a frequency dependence of the Rayleigh length $z_{R}(\omega)$, which determines $\varphi_{G}(z, \omega)$ and hence $\phi(z)$ as well as the electric field envelope $\widetilde{E}(t)$. Closely following the discussion and the nomenclature of Ref. 7, we can identify three special cases: (i) frequencyindependent beam width in front of the lens, ${ }^{18}$ (ii) 
frequency-independent Rayleigh length, ${ }^{19}$ and (iii) frequency-independent beam waist in the focus. ${ }^{20}$ To obtain simple analytical results, we employ two approximations: First, we assume that the deviations of phase velocity $v_{\text {phase }}$ and group velocity $v_{\text {group }}$ from vacuum velocity of light $c_{0}$ are small, allowing for Taylor expansions. The validity of this approximation follows from the Fresnel approximation made above. Second, we assume that the envelope of the pulse remains undistorted. In this limit the group velocity at the carrier frequency describes the velocity of the envelope. With this it is straightforward to arrive at the general form for the carrier-envelope phase:

$$
\begin{aligned}
\phi(z) & =\frac{\omega_{0}}{c_{0}^{2}} \int_{-\infty}^{z}\left[v_{\text {phase }}\left(z^{\prime}\right)-v_{\text {group }}\left(z^{\prime}\right)\right] \mathrm{d} z^{\prime} \\
& =\varphi_{G}\left(z, \omega_{0}\right)-\omega_{0} \frac{\partial \varphi_{G}}{\partial \omega}\left(z, \omega_{0}\right) .
\end{aligned}
$$

Upon working out the derivative we obtain

$$
\phi(z)=\arctan (Z)+\mathcal{F} \frac{Z}{1+Z^{2}},
$$

with the abbreviation $Z=z / z_{R}\left(\omega_{0}\right)$. Factor $\mathcal{F}$ is given by $\mathcal{F}=-1$ for case (i), $\mathcal{F}=0$ for case (ii), and $\mathcal{F}=+1$ for case (iii). More generally, this expression for $\phi(z)$ remains valid even for arbitrary noninteger values of $\mathcal{F}$, in which case $\mathcal{F}$ is defined by the scaling

$$
z_{R}(\omega) \propto \omega^{\mathcal{F}} .
$$

The scalings for the beam waist, $w_{0}(\omega) \propto \omega^{+(\mathcal{F}-1) / 2}$, and the beam width at the lens, $w\left(z_{f}, \omega\right) \propto \omega^{-(\mathcal{F}+1) / 2}$, follow.

The three solid curves in Fig. 2(b) illustrate cases (i)-(iii). Only for case (ii), where $v_{\text {group }}(z)=c_{0}$, is the carrier-envelope phase identical to the Gouy phase shift, i.e., $\phi(z)=\varphi_{G}\left(z, \omega_{0}\right)$. For case (i), $\phi(z)$ has a horizontal slope in the focus because $v_{\text {group }}(z=0)$ $=v_{\text {phase }}(z=0)>c_{0}$. For case (iii) the slope of $\phi(z)$ in the focus is steeper than in case (ii) as $v_{\text {group }}(z=0)<c_{0}$ while $v_{\text {phase }}(z=0)>c_{0}$.

Experimentally, it is usually not easy to specify the frequency dependence of the beam width $w\left(z_{f}, \omega\right)$ in front of the lens because it results from rather complex processes within the mode-locked laser oscillator. It would be surprising if this frequency dependence turned out to be negligible [case (i)]. Our experiment lies between cases (ii) and (iii).

In conclusion, we have realized a simple solidstate-based technique suitable for measuring the variation of the carrier-envelope phase of few-cycle laser pulses in the focus of a lens arising from the topological Gouy phase. The approach is based on the well-known $f$-to- $2 f$ self-referencing. Importantly, we have generated the corresponding beat signal in a re- gion thinner than the Rayleigh length, such that the local carrier-envelope phase can be measured. Furthermore, unlike other approaches based on multiphoton ionization of atoms, ${ }^{10}$ our approach works with pulses directly from a usual $81-\mathrm{MHz}$ modelocked laser oscillator. We have also shown by simple analytical formulas that the evolution of the carrierenvelope phase critically depends on the frequency dependence of the laser beam profile. This frequency dependence is expected to be affected not only by the laser system but also by its actual alignment. Our experimental approach allows for a simple routine characterization of the resulting local carrierenvelope phase. In the emerging field of extreme nonlinear optics, such knowledge concerning the carrierenvelope phase is crucial.

The work of M. Wegener has been supported by project DFG-We 1497/9-1. We thank F. X. Kärtner and U. Morgner for providing the double-chirped mirrors. T. Tritschler's e-mail address is thorsten. tritschler@physik.uni-karlsruhe.de.

\section{References}

1. C. R. Gouy, Acad. Sci. Paris C. R. 110, 1251 (1890).

2. C. R. Gouy, Ann. Chim. Phys. 24, 145 (1891).

3. A. E. Siegmann, Lasers (University Science, Mill Valley, Calif., 1986); note that the sign convention used for the Gouy phase in this textbook is different from the one used in this Letter.

4. D. Subbarao, Opt. Lett. 20, 2162 (1995).

5. M. V. Berry, Proc. R. Soc. London Ser. A 392, 45 (1984).

6. S. Feng and H. G. Winful, Opt. Lett. 26, 485 (2001).

7. M. Wegener, Extreme Nonlinear Optics (SpringerVerlag, Berlin, 2004).

8. A. B. Ruffin, J. V. Rudd, J. F. Whitaker, S. Feng, and H. G. Winful, Phys. Rev. Lett. 83, 3410 (1999).

9. E. Budiarto, N.-W. Pu, S. Jeong, and J. Bokor, Opt. Lett. 23, 213 (1998).

10. F. Lindner, G. G. Paulus, H. Walther, A. Baltuška, E. Goulielmakis, M. Lezius, and F. Krausz, Phys. Rev. Lett. 92, 113001 (2004).

11. U. Morgner, F. X. Kärtner, S. H. Cho, Y. Chen, H. A. Haus, J. G. Fujimoto, E. P. Ippen, V. Scheuer, G. Angelow, and T. Tschudi, Opt. Lett. 24, 411 (1999).

12. O. D. Mücke, T. Tritschler, M. Wegener, U. Morgner, and F. X. Kärtner, Opt. Lett. 27, 2127 (2002).

13. D. J. Jones, T. M. Fortier, and S. T. Cundiff, J. Opt. Soc. Am. B 21, 1098 (2004).

14. X. W. Sun and H. S. Kwok, J. Appl. Phys. 86, 408 (1999)

15. O. D. Mücke, T. Tritschler, M. Wegener, U. Morgner, and F. X. Kärtner, Phys. Rev. Lett. 89, 127401 (2002).

16. S. T. Cundiff, W. H. Knox, E. P. Ippen, and H. A. Haus, Opt. Lett. 21, 662 (1996).

17. T. M. Fortier, D. J. Jones, and S. T. Cundiff, Opt. Lett. 28, 2198 (2003).

18. Z. L. Horváth, J. Vinkó, Zs. Bor, and D. von der Linde, Appl. Phys. B: Lasers Opt. 63, 481 (1996).

19. S. Feng, H. G. Winful, and R. W. Hellwarth, Phys. Rev. E 59, 4630 (1999).

20. A. E. Kaplan, J. Opt. Soc. Am. B 15, 951 (1998). 